\title{
Modelling of Rotary EDM Process Parameters of Inconel 718 using Artificial Neural Networks
}

\author{
Jayaraj JEEVAMALAR*, Sundaresan RAMABALAN**, Chinnamuthu SENTHILKUMAR*** \\ *E.G.S. Pillay Engineering College, Nagapattinam, Tamilnadu, India, E-mail: J.Jeevamalar@gmail.com \\ **E.G.S. Pillay Engineering College, Nagapattinam, Tamilnadu, India, E-mail: cadsrb@gmail.com \\ ***Annamalai University, Chidambaram,Tamilnadu, India,E-mail: csmfg_au@yahoo.com \\ cross $^{\text {ref }}$ http://dx.doi.org/10.5755/j01.mech.26.6.20484
}

\section{Introduction}

Nowadays the researchers utilize Artificial Neural Networks (ANN) for demonstrating complex modern industrial issues. ANN is a great alternative to traditional experiential modelling primarily based on polynomial and linear regressions [1]. Utilizing ANNs would prompt sparing time and cost by conceiving the experiential results [2]. Recently, ANNs have become a predominant method in the modelling of manufacturing-related complex issues because of their capacity to learn and to generalize (interpolate) the unpredictable relations among input and output parameters [3]. ANN avoids the limitations of the conventional methods by extracting the desired information using the input data [4-5]. Furthermore, ANN's have model-free estimators, i.e. they can model complex input-output association without using any scientific model [6].

ANN was configured with different layers, and thus named as multilayer ANNs. Multilayer Perceptron (MLP) Neural Networks comprises an input, hidden and output layers. The best possible number of neurons within the in the hidden layer was identified by a series of network configurations, during which the number of neurons varied from 1 to 30 . The Root Means Square Error (RMSE) was considered as the error function. Generally, a technique of trial and error was used to define the number of neurons (nodes) within the hidden layer. Many endeavors are taken to research network performance by varying the number of hidden nodes. Therefore, different candidate networks were configured, each was trained independently, and therefore the best network was chosen in keeping with the accuracy of the estimates within the testing phase. It should be noted that if the number of hidden nodes is excessively huge, the ANN could be over-trained to offer false values within the testing phase. If too few nodes are selected, the proper relation might not be achieved due to the training of the neurons. The data acquired from the experiments were applied to train the network. The scaled data were passed into the input layer and then they were disseminated from the input layer to the output layer through hidden layers. Every node in a hidden or output layer was served as a summing point to combine and modify the inputs from the preceding layer. The training is the process by which the measures and biases are adjusted efficiently so that the network shows some anticipated performance. To achieve a supervised training, ANN output error might be evaluated.

As modelling of a process decreases the effort, cost and time for optimal and efficient application of that process, it has a noteworthy role in the Electrical Dis- charge Machining (EDM) process modelling also. Several researchers have been carried out in this direction using ANN modelling, but still, they need more enhancements. So to find the direction of enhancement, a literature review has been carried out as follows: Juhr et al. [7] compared the experimental results of a nonlinear regression model and the ANN model for the generation of continuous parameter technology. It was found that the ANN exhibits improved prediction accuracy than the proposed regression model. Panda \& Bhoi [8] formulated an ANN model using the Levenberg-Marquardt learning algorithm and logistic sigmoid transfer function to estimate the Material Removal Rate (MRR). It was reported that a Feed Forward Neural Networks (FFNN) model with a 3-7-1 network configuration delivers quicker and more accurate results. Markopoulos et al. [9] applied an ANN model for the prediction of Surface Roughness (SR) using Matlab ${ }^{\circledR}$ and Netlab ${ }^{\circledR}$. It was reported that both simulators were found effective for the calculation of SR. Joshi \& Pande [10] proposed two models using the Finite Element Method (FEM) and ANN for the advanced machining process. An optimal ANN model with the network configuration of 4-8 $-12-4$ was achieved to provide very good prediction accuracies for MRR, crater depth and crater radius and a reasonable one for Tool Wear Rate (TWR). Thillaivannan et al. [11] created an ANN model to optimize the input factors with the minimum machining time using the Taguchi technique. Feed Forward Back Propagation Networks (FFBPN) with two Back Propagation (BP) algorithms, namely Gradient descent and gradient descent with momentum, were used for finding a correlation between the target performance measure and input factors. Fenggou \& Dayong [12] proposed an ANN modelling technique to find the number of hidden nodes and optimize the correlation between input variables and performance measures using Genetic Algorithm (GA) and Back Propagation Learning Algorithm (BPLA). It was reported that 8 hidden nodes were observed to be ideal for a required dimensional accuracy and performance. Khan et al. [13] developed an ANN model with MLP neural architecture to predict the value of SR on Ti-15-3 alloy. An average of $6.15 \%$ error was observed between required and predicted values of SR which found to be in good tradeoff with the test results. Panda [14] developed a novel hybrid method of the NeuroGrey Modeling (NGM) method. This system has been examined for the optimization of multiple parameters (e.g. SR, Micro-Hardness (MH), the thickness of the recast layer and MRR) of the process. In this approach, R-square and Means Square Error (MSE) are used to evaluate the efficiency of ANNs. 
Several researchers reported that the Back Propagation algorithm is the most appropriate approach for handling large learning problems. Based on the structure of an ANN in a problem solution, they can be classified into two types: (i) Feedback Neural Networks (FBNN) and (ii) Feed Forward Neural Networks (FFNN). The FBNNs have widely used an algorithm due to their simple structure and ability to analyze the problem mathematically. The Back Propagation Neural Networks (BPNN) scheme has mathematically strong learning ability in training and relating input and output variables [15]. They are usually called feed-forwarded, multi-layered networks. The multilayer preceptor trained BP algorithm has been observed very successfully in this research.

In the FFNN model, each layer comprises a set of nodes that share an equivalent input and output links, but that does not connect with the other nodes in the same layer. Moreover, links are strictly concerned with a directed graph: from the input to the output. Using a sigmoid transfer function for the hidden layer and linear transfer function for the output layer are known to be universal approximates. Mathematically training a network is supposed to minimize the error of a cost function, such as the Mean Squared Error function. A commonly used performance function is that the MSE which tries to minimize the average squared error between the networks output and thus the output value over all the instance pairs.

This research considers the factors, which could affect the efficiency of the ANN model established, according to the item required by the Matlab 2009 toolbox to develop the ANN model. Each input node denotes an input parameter while the output neurons provide the dependent response. Hidden layers are used to perform nonlinear transformations on the input space and are used for computation purposes.

\section{Design of experts}

Workpieces of sliced into $3 \mathrm{~mm}$ thickness from $25 \mathrm{~mm}$ diameter rod of Inconel 718 using wire cut EDM. ELEKTRA M100 die sinking machine was used for experimentation. The specification of this machine is max working current - 25 Amp, work table size -550 x $350 \mathrm{~mm}$, Power supply-3 phase, 440V, 30-50 Hz. Table 1 gives details of the experimental design. MRR and average Surface Roughness are considered as output responses.

Table 1

Experimental levels

\begin{tabular}{|c|c|c|c|c|c|}
\hline Sl. No. & Process parameters & Unit & \multicolumn{3}{|c|}{ Levels } \\
\hline 1 & Peak current $I_{p}$ & Amp & 10 & 12.5 & 15 \\
\hline 2 & Pulse-on time $T_{\text {on }}$ & $\mu \mathrm{s}$ & 500 & 1000 & 1500 \\
\hline 3 & Pulse-off time $T_{\text {off }}$ & $\mu \mathrm{s}$ & 200 & 500 & 800 \\
\hline
\end{tabular}

In this study, 20 electrodes are selected for experiments. The tools of $12 \mathrm{~mm}$ exterior and $9 \mathrm{~mm}$ interior diameters, made up of copper material were made using conventional machining techniques and is shown in Fig. 1.

From the detailed from the literature, it's clear that gaining the specified level of Material Removal Rate (MRR) and Surface Roughness (SR) is a challenging task for super alloys. Therefore, during this study, MRR and SR were identified as the output responses. MRR is calculated based on the weight before and after the machining of the workpieces. Machining time was measured employing a stopwatch. Roughness was calculated at three different randomly selected locations and average SR values are considered. The experiments were performed by $\mathrm{L}_{20}$ Orthogonal Array.

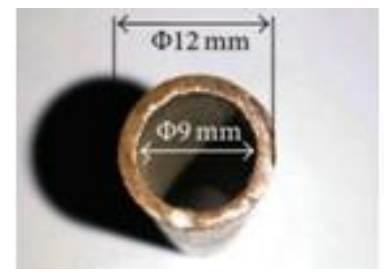

Fig. 1 Photographic image of the tool

\section{ANN model description}

ANN is a flexible computing technique which can train and correlate input-output factors to resolve intricate non-linear problems. The ANN validation has been established using the NN toolbox of Matlab 2009 software. To train the network, a multilayer Feed-Forward Network with Back Propagation (BPNN) algorithm was used. In the present method, ANN is used for solving non-linear model which is easy to use and apprehend as compared to statistical techniques. ANN with BPLA is extensively utilized in solving numerous classifications and forecasting problems.

Modelling of Electrical Discharge Drilling (EDD) processes with BPNN consists of three stages: training, testing and validation of the networks with experimental machining data. The training data contains values for $I_{p}$ (A), $T_{o n}(\mathrm{~B})$ and $T_{\text {off }}(\mathrm{C})$ and therefore the associated outputs are MRR and SR. Total 20 sets of data were used, out of which 14 were selected randomly and used for training purposes whereas the remaining 3 data sets were used for testing and 3 datasets were used for validation purposes. Before modelling, the data might be trained and therefore the mapping to be understood. It is important to process the experimental data into patterns. The training/testing pattern vectors were moulded and formed with an input condition vector $P_{i}$ and therefore the corresponding target vector, $T_{i}$.

$$
P_{i}=\left[\begin{array}{l}
\text { Peak Current }(\mathrm{A}) \\
\text { Pulse }- \text { on time }(\mathrm{B}) \\
\text { Pulse }- \text { off time }(\mathrm{C})
\end{array}\right]
$$

$$
T_{i}=\left[\begin{array}{l}
\text { Material Removal Rate (MRR) } \\
\text { Surface Roughness (SR) }
\end{array}\right]
$$

A trial and error method has got to be used for estimating the number of nodes within the hidden layer. As such, efforts have been taken to analyze network performance with a distinct number of hidden nodes.

Therefore, some of the candidate networks are configured each trained independently, and therefore the optimum one was chosen according to the accuracy of the estimates in the testing phase. It should be observed that if the number of hidden nodes is just too large, the ANN, could be over-trained giving false values within the testing phase if too few nodes are selected, the mapping won't be achieved due to under-training of three nodes (i.e. for $I_{p}$, $T_{o n}$ and $\left.T_{o f f}\right)$. Two nodes within the output layer represent 
the output responses (i.e. for MRR and SR). The data used for training the ANN model are tabulated in Table 2.

Table 2

ANN model data for Rotary tool EDD

\begin{tabular}{|c|c|}
\hline Name & ANN model \\
\hline Network type & FFBP model \\
\hline Number of nodes hidden layers & 7 \\
\hline Transfer function & PURELIN \\
\hline Training function & TRAINLM \\
\hline Learning function & LEARNGDM \\
\hline Performance function & Mean square error \\
\hline Number of nodes & 7 \\
\hline SS error & 0.03405 \\
\hline Number of epochs & 9 \\
\hline Validation checks & 5 \\
\hline Learning factor & 0.6 \\
\hline
\end{tabular}

The ANN has trained with Levenberg-Marquardt backpropagation algorithm (TRAINLM). The input layer consists of 3 nodes for three decision variables of the study (i.e. $I_{p}, T_{o n}$ and $T_{o f f}$ ). The hidden layer consists of 7 nodes and the output layer consists of two nodes for MRR and SR.

The consistency of the built ANN is highly dependable not only on the algorithm of ANN training and its parameters but also on several architectural parameters of ANN. Above all, theoretical and practical context is restricted to help with the systematic selection of ANN parameters through the entire development and training phase of ANN. Because of this, ANN parameters are usually set by an extremely time-consuming experience in trial and error procedures. In this way, the optimal settings of ANN parameters are not guaranteed to achieve the best quality. The ANN training module is shown in Fig. 2.

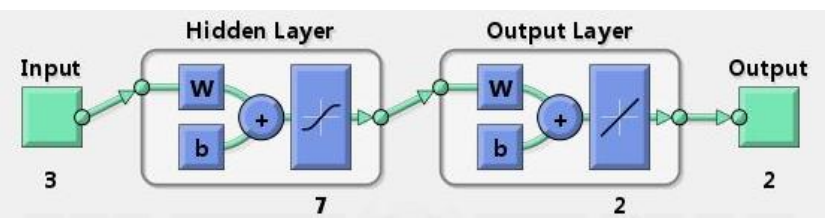

Fig. 2 ANN training module

To have the maximum efficient ANN simulation model, seven model architectures had been tested for every set of input parameters. The number of neurons inside the hidden layer was varied from 2-9. The performance and regression graphs for MRR and SR obtained using rotary tool EDD are presented in Figs. 3 and 4 correspondingly. Successful training was realized after 9 iterations with MSE error of 0.03405 and 5 validating checks. The Rvalue for the training data was at 0.99978 and the R-value for the testing data was 0.86677 for rotary tool EDD.

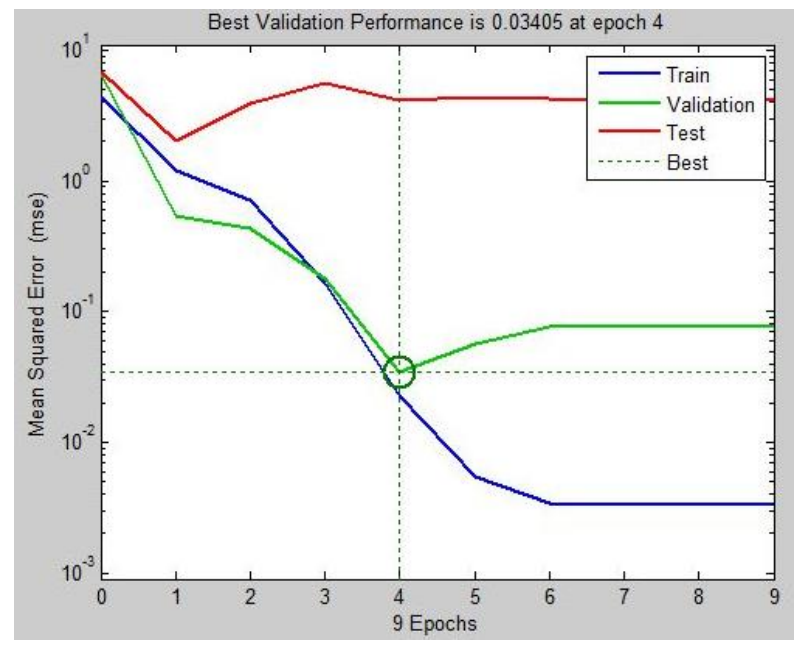

Fig. 3 Performance curve
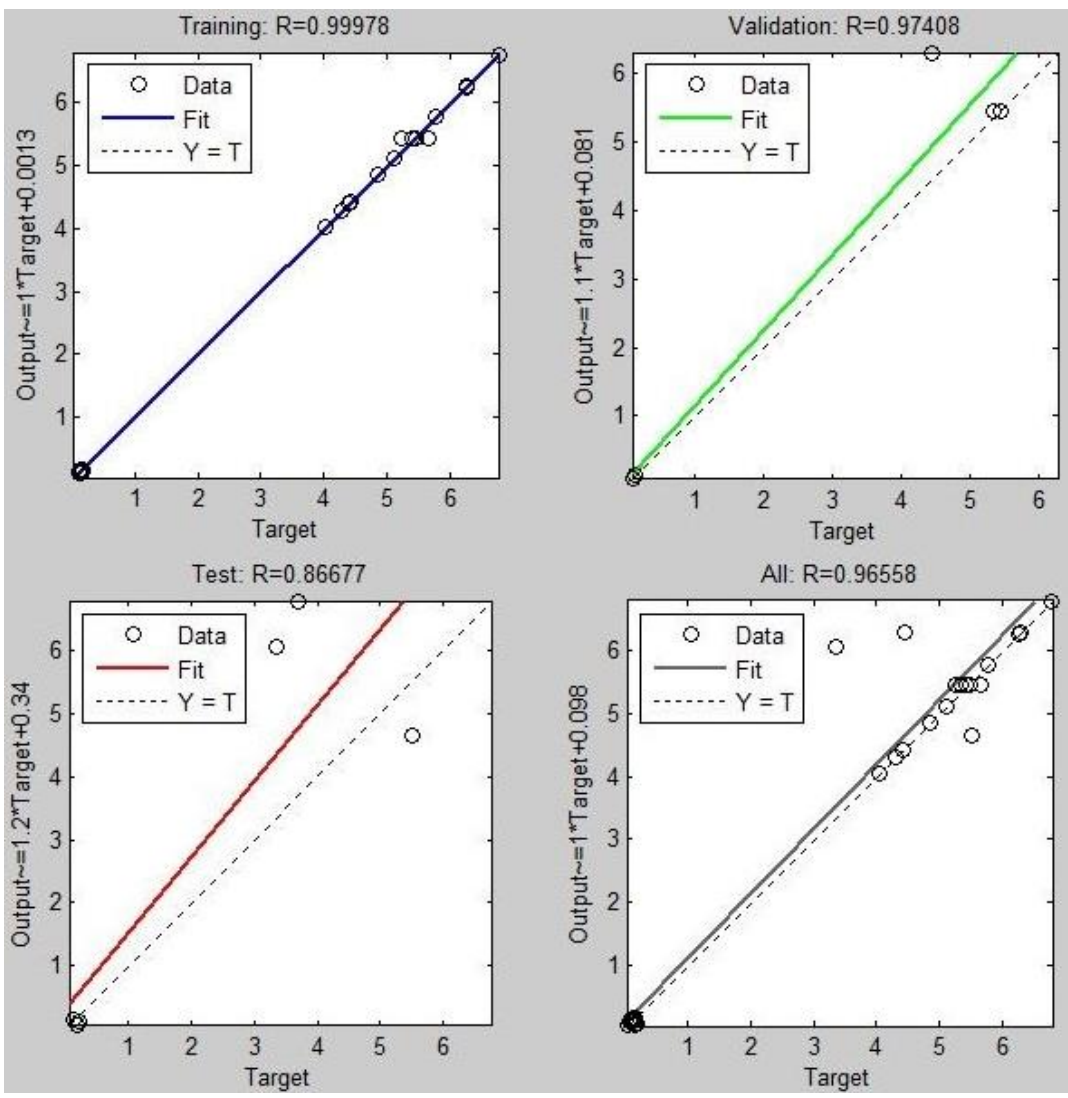

Fig. 4 Regression Plots 


\section{Verification of trained networks}

Table 3 compares the experimental results and ANN predictions concerning MRR and SR for the rotary tool EDD as well as given the error. It was found that the ANN model shows a good agreement between the actual and the predicted results.

Table 3

Comparison of experimental and ANN values

\begin{tabular}{|c|c|c|c|c|c|c|}
\hline \multirow{2}{*}{ Runs } & \multicolumn{2}{|c|}{ MRR, g./min. } & \multirow{2}{*}{ Error } & \multicolumn{2}{|c|}{ SR, $\mu \mathrm{m}$} & \multirow{2}{*}{ Error } \\
\cline { 2 - 3 } \cline { 5 - 6 } & Exp. & Pred. & & Exp. & Pred. & \\
\hline 1 & 0.042 & 0.043 & -0.001 & 4.845 & 4.827 & 0.018 \\
\hline 2 & 0.065 & 0.064 & 0.001 & 4.452 & 4.731 & -0.279 \\
\hline 3 & 0.167 & 0.165 & 0.002 & 4.036 & 4.004 & 0.032 \\
\hline 4 & 0.211 & 0.206 & 0.005 & 5.503 & 5.32 & 0.183 \\
\hline 5 & 0.174 & 0.157 & 0.017 & 3.651 & 3.976 & -0.325 \\
\hline 6 & 0.163 & 0.173 & -0.01 & 5.108 & 5.291 & -0.183 \\
\hline 7 & 0.221 & 0.208 & 0.013 & 4.582 & 4.792 & -0.21 \\
\hline 8 & 0.263 & 0.251 & 0.012 & 6.269 & 5.744 & 0.525 \\
\hline 9 & 0.152 & 0.162 & -0.01 & 4.406 & 4.196 & 0.21 \\
\hline
\end{tabular}

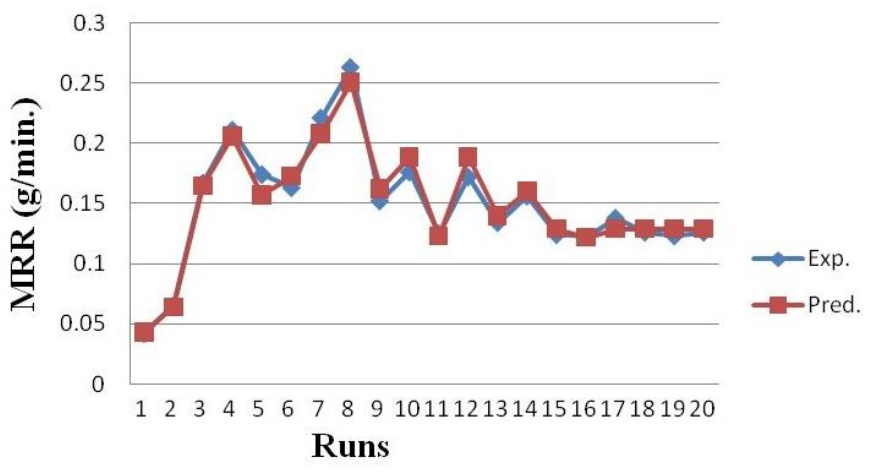

Fig. 5 Comparison of experimental and ANN outputs on MRR

\section{Conclusions}

In the current work, the ANN model has been developed for the EDM drilling process for machining of Inconel 718 with the hollow tubular copper electrode. The training and testing of ANN for input-output patterns are administered by through Neural Network Toolbox in Matlab 2009 software package. An ANN was selected, trained, tested, and validated, then implemented for the simulation to reinforce MRR and SR. From the experimental investigation, the subsequent conclusions are derived.

1. The results of the ANN model showed close matching between the model outputs and thus the measured outputs. It had been understood that the performance of ANN in mapping nonlinear relationships among inputs and outputs was good. The estimated machining output with the actual machining performance and a good performance agreement was obtained.

2. From Table 3, it is clear that the maximum error is 0.017 for experiment number 5 ; the minimum is 0.00 for experiment number 16 for MRR. In the case of SR, the maximum error is 0.525 for experiment number 8 ; the minimum is 0.018 for experiment number 1 . It had been observed that the calculated error was within the permissible limit range of $\pm 10 \%$. Obtained results revealed a good trade-off between experimentation and ANN predictions.

\begin{tabular}{|c|c|c|c|c|c|c|}
\hline \multirow{2}{*}{ Runs } & \multicolumn{2}{|c|}{ MRR, g./min. } & \multirow{2}{*}{ Error } & \multicolumn{2}{c|}{ SR, $\mu \mathrm{m}$} & \multirow{2}{*}{ Error } \\
\cline { 2 - 6 } & Exp. & Pred. & & Exp. & Pred. & \\
\hline 10 & 0.176 & 0.189 & -0.013 & 5.768 & 5.376 & 0.392 \\
\hline 11 & 0.125 & 0.123 & 0.002 & 6.454 & 6.228 & 0.226 \\
\hline 12 & 0.172 & 0.189 & -0.017 & 3.38 & 3.72 & -0.34 \\
\hline 13 & 0.134 & 0.14 & -0.006 & 3.684 & 3.645 & 0.039 \\
\hline 14 & 0.156 & 0.16 & -0.004 & 4.42 & 4.402 & 0.018 \\
\hline 15 & 0.124 & 0.129 & -0.005 & 5.417 & 5.408 & 0.009 \\
\hline 16 & 0.122 & 0.122 & 0.000 & 5.347 & 5.408 & -0.061 \\
\hline 17 & 0.138 & 0.129 & 0.009 & 5.47 & 5.408 & 0.062 \\
\hline 18 & 0.126 & 0.129 & -0.003 & 5.243 & 5.408 & -0.165 \\
\hline 19 & 0.123 & 0.129 & -0.006 & 5.651 & 5.408 & 0.243 \\
\hline 20 & 0.126 & 0.129 & -0.003 & 5.468 & 5.408 & 0.06 \\
\hline
\end{tabular}

The comparison of the experimental results and the ANN predictions of MRR and SR for the rotary tool electrical discharge drilling process are shown in Figs. 5 and 6 . These figures show a close relationship between these two output performances. Hence, the ANN is capable of predicting the performance measures for the given range of input parameters.

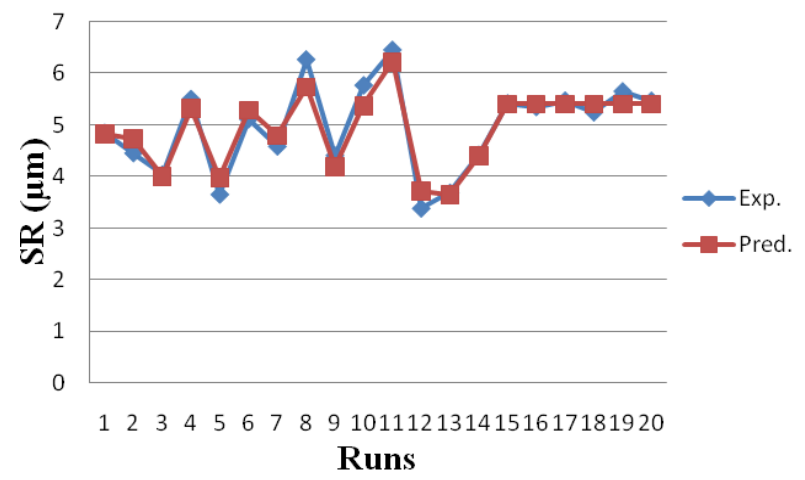

Fig. 6 Comparison of experimental and ANN outputs on SR

\section{References}

1. Kose, E. 2008. Modeling of colour perception of different age groups using artificial neural networks, Journal of Expert Systems with Applications 34(3): 21292139.

https://doi.org/10.1016/j.eswa.2007.02.036.

2. Shabanzadeh, P.; Senu, N.; Shameli, K.; Ismail, F.; Mohagheghtabar, M. 2013. Application of artificial neural network (ANN) for the prediction of size of silver nanoparticles prepared by green method, Digest Journal of Nanomaterials and Biostructures 8(2): 541549.

3. Freeman, J. A.; Skapura, D. M. 1991. Neural networks: algorithms, applications, and programming techniques. Addison-Wesley Publishing Company, New York.

4. Mandal, S.; Sivaprasad, P. V.; Venugopal, S.; Murthy, K. P. N. 2009. Artificial neural network modelling to evaluate and predict the deformation behavior of stainless steel type AISI 304L during hot torsion, Journal of Applied Soft Computing 9(1): 237-244. https://doi.org/10.1016/j.asoc.2008.03.016.

5. Akcayol, M. A.; Cinar, C. 2005. Artificial neural network based modeling of heated catalytic converter performance, Journal of Applied Thermal Engineering 25: 2341-2350.

https://doi.org/10.1016/j.applthermaleng.2004.12.014. 
6. Fausett, L. 1994. Fundamentals of neural networks: architectures, algorithms, and applications. PrenticeHall, Inc., USA.

7. Juhr, H.; Kunanz, K.; Nestler, A.; Leitte, G. 2004. Generation of parameter technologies for EDM die sinking with Artificial Neural Networks (ANN) and Nonlinear Regression Functions (NRF), Forschungsergebnisbericht.

8. Panda, D. K.; Bhoi, R. K. 2007. Artificial neural network prediction of material removal rate in electro discharge machining, Journal of Materials and Manufacturing Processes 20(4): 645-672. https://doi.org/10.1081/AMP-200055033.

9. Markopoulos, A. P.; Manolakos, D. E.; Vaxevanidis, N. M. 2008. The Artificial neural network models for the prediction of surface roughness in electrical discharge machining, Journal of Intelligent Manufacturing 19: 283-292. https://doi.org/10.1007/s10845-008-0081-9.

10. Joshi, S. N.; Pande, S. S. 2009. Development of an intelligent process model for EDM, International Journal of Advanced Manufacturing Technology 45: 300317. https://doi.org/10.1007/s00170-009-1972-4.

11. Thillaivanan, A.; Asokan, P.; Srinivasan, K. N.; Saravanan, R. 2010. Optimization of operating parameters for the EDM process based on Taguchi method and Artificial Neural Network, International Journal of Engineering Science and Technology 2(12): 68806888.

12. Fenggou, C.; Dayong, Y. 2004. The study of high efficiency and intelligent optimization system in EDM sinking process, Journal of Materials Processing Technology 149: 83-87.

https://doi.org/10.1016/j.jmatprotec.2003.10.059.

13. Khan, M. A. R.; Rahman, M. M.; Kadirgama, K.; Maleque, M. A.; Bakar, R. A. 2011. Artificial intelligence model to predict surface roughness of Ti-15-3 alloy in EDM Process, International Journal of Mechanical and Mechatronics Engineering 5(2): 503-507. https://doi.org/10.5281/zenodo.1061591

14. Panda, D. K. 2010. Modelling and optimization of Multiple Process attributes of electro discharge machining process by using a new hybrid approach of nuero- grey modeling, Journal of Materials and Manufacturing Processes 25(6): 450-461. https://doi.org/10.1080/15394450902996551.

15. Jain, R. K.; Jain, V. K.; Kalra, P. K. 1999. Modelling of abrasive flow machining process: a neural network approach, Journal of Wear 231(2): 242-248. https://doi.org/10.1016/S0043-1648(99)00129-5.

\section{J. Jeevamalar, S. Ramabalan, C. Senthilkumar \\ MODELLING OF ROTARY EDM PROCESS PARAMETERS OF INCONEL 718 USING ARTIFICIAL NEURAL NETWORKS}

S u m m a r y

This paper explains the methodological procedure and the outcome of the Artificial Neural Networks modelling process for the rotary Electrical Discharge Machining of Inconel 718. The important process parameters during this work are Peak Current, Pulse-on time and Pulse-off time with machining performances of Material Removal Rate and Surface Roughness. In such conditions, an Artificial Neural Networks model was developed using Matlab toolbox and Feed Forward Back Propagation technique was used to predict the responses. The investigational data were separated to train, test the network and validate the model. The developed model has been confirmed experimentally for training and testing by considering the number of iterations and Mean Square Error convergence criteria. The developed model results are used to approximate the responses fairly exactly. The model features a mean coefficient of correlation of 0.96558 . Results exposed that the projected model is employed for the prediction of the complex EDM process.

Keywords: Electrical discharge machining, Inconel 718, Artificial neural network, Material removal rate, Surface roughness.

Received April 30, 2018

Accepted December 01, 2020

This article is an Open Access article distributed under the terms and conditions of the Creative Commons Attribution 4.0 (CC BY 4.0) License (http://creativecommons.org/licenses/by/4.0/). 